Article

\title{
Inverse Derivative Operator and Umbral Methods for the Harmonic Numbers and Telescopic Series Study
}

\author{
Giuseppe Dattoli ${ }^{1,+}$, Silvia Licciardi ${ }^{1, *,+}$ (1) and Rosa Maria Pidatella ${ }^{2,+}$ \\ 1 ENEA-Frascati Research Center, Via Enrico Fermi 45, 00044 Rome, Italy; pinodattoli@libero.it \\ 2 Department of Mathematics and Computer Science, University of Catania, Viale A. Doria 6, \\ 95125 Catania, Italy; rosa@dmi.unict.it \\ * Correspondence: silviakant@gmail.com; Tel.: +39-392-509-6741 \\ + The authors contributed to this work according to what declared in the Author Contribution Section.
}

Citation: Dattoli, G.; Licciardi, S.; Pidatella, R.M. Inverse Derivative Operator and Umbral Methods for the Harmonic Numbers and Telescopic Series Study. Symmetry 2021, 13, 781. https://doi.org/ $10.3390 /$ sym13050781

Academic Editor: Paolo Emilio Ricci and Charles F. Dunkl

Received: 26 March 2021

Accepted: 29 April 2021

Published: 1 May 2021

Publisher's Note: MDPI stays neutral with regard to jurisdictional claims in published maps and institutional affiliations.

Copyright: (c) 2021 by the authors. Licensee MDPI, Basel, Switzerland. This article is an open access article distributed under the terms and conditions of the Creative Commons Attribution (CC BY) license (https:// creativecommons.org/licenses/by/ $4.0 /)$.

\begin{abstract}
The formalism of differ-integral calculus, initially developed to treat differential operators of fractional order, realizes a complete symmetry between differential and integral operators. This possibility has opened new and interesting scenarios, once extended to positive and negative order derivatives. The associated rules offer an elegant, yet powerful, tool to deal with integral operators, viewed as derivatives of order-1. Although it is well known that the integration is the inverse of the derivative operation, the aforementioned rules offer a new mean to obtain either an explicit iteration of the integration by parts or a general formula to obtain the primitive of any infinitely differentiable function. We show that the method provides an unexpected link with generalized telescoping series, yields new useful tools for the relevant treatment, and allows a practically unexhausted tool to derive identities involving harmonic numbers and the associated generalized forms. It is eventually shown that embedding the differ-integral point of view with techniques of umbral algebraic nature offers a new insight into, and the possibility of, establishing a new and more powerful formalism.
\end{abstract}

Keywords: umbral methods 05A40; operators theory 44A99, 47B99, 47A62; special functions 33C52, 33C65, 33C99, 33B10, 33B15; integral calculus 97I50; harmonic numbers 05A99, 11B75; combinatorics 05A10, 11B75; gamma function 33B15; telescopic series 11B65, 11B75, 05A10

\section{Introduction}

New concepts and techniques emerged in the past within the framework of special functions have had positive feedback in other and more abstract fields of Mathematics. The techniques associated with umbral calculus have opened new and unexpected avenues in analysis and simplified the technicalities of calculations, which are awkwardly tedious when performed with conventional computational means. They, furthermore, have allowed a general principle of symmetry between special functions, providing a method of establishing a formal equivalence between different families of functions (e.g., Bessel and Gaussian functions).

The Authors of this paper have largely benefitted from the techniques suggested by umbral methods and have embedded them with other means associated, e.g., with algebraic operational procedure, to obtain new results and to reformulate previous, apparently extraneous topics, within a unifying point of view.

This paper follows the same stream. We embed umbral methods and formal integration techniques to explore the field of number series by getting a non-conventional treatment of problems usually treated with completely different means. We will recover new and old results. Yet, the merits of this paper may rely on the novelty and generality of the method itself.

We develop a new framework to treat problems in Number Theory, Combinatorial Analysis, Telescopic Series, Harmonic Numbers... The method we foresee gathers together integro-differential and umbral means. The paper consists of two parts, the first is devoted 
to the formalism of Negative Derivative Operator and to the relevant use for the previously quoted fields of research. In the second part, we discuss the application of umbral methods and how they interface with the integro-differential counterpart.

Elementary problems in calculus reveal unexpected new features if viewed from a broader perspective which employs, e.g., operational methods. The use of the negative derivative operator formalism [1,2] has provided an efficient tool to compute the primitive of a given function, or of products of functions as well. The underlying formalism allows the handling of integrals and derivatives on the same footing. Within this framework the primitive of the product of two functions is nothing but a restatement of the Leibniz formula [3,4] as it has been proved in ref. [5], namely

Definition 1. $\forall x \in \operatorname{Dom}\{f, g\}$ we state

$$
\hat{D}_{x}^{-1}(g(x) f(x))=\sum_{s=0}^{\infty}\left(\begin{array}{c}
-1 \\
s
\end{array}\right) g^{(-1-s)}(x) f^{(s)}(x)
$$

where

$$
\hat{D}_{x}^{-1} s(x)=\int s(x) d x
$$

is the negative derivative operator, $f^{( \pm s)}(x)$ denotes the $s^{\text {th }}$-(positive/negative)—derivative of the function, and

$$
\left(\begin{array}{c}
-1 \\
s
\end{array}\right)=(-1)^{s}
$$

For the operation of definite integration, we set

$$
{ }_{\alpha} \hat{D}_{x}^{-1} s(x)=\int_{\alpha}^{x} s(\xi) d \xi=S(x)-S(\alpha) .
$$

By exploiting Definition 1, we state what follows.

Proposition 1. The integral of a function $f \in C^{\infty}$ can be written in terms of the series

$$
F(x):=\int f(x) d x=\sum_{s=0}^{\infty}(-1)^{s} \frac{x^{s+1}}{(s+1) !} f^{(s)}(x), \quad \forall x \in \operatorname{Dom}\{f\},
$$

where $f^{(s)}(x)$ denotes the $s^{\text {th }}$-derivative of the integrand function.

Proof. We write Equation (5) according to Definition 1 as (to simplify the writing, we will use $\hat{D}_{x}^{-1}={ }_{0} \hat{D}_{x}^{-1}$ to treat integrals with lower extreme of integration $\alpha=0$ ) $\hat{D}_{x}^{-1}(g(x) f(x))=\sum_{s=0}^{\infty}\left(\begin{array}{c}-1 \\ s\end{array}\right) g^{(-1-s)}(x) f^{(s)}(x)$. By assuming $g(x)=1$, we get

$$
g^{(-1-s)}(x)=\hat{D}_{x}^{(-1-s)} 1=\frac{x^{s+1}}{(s+1) !}
$$

thus, eventually ending up with

$$
\int f(x) d x=\hat{D}_{x}^{-1}(1 \cdot f(x))=\sum_{s=0}^{\infty}(-1)^{s} \frac{x^{s+1}}{(s+1) !} f^{(s)}(x) .
$$

For further comments, the reader is directed to refs. [1,2,5].

Example 1. Let us now consider the primitive of the unit function and write

$$
\int 1 d \xi=\int \xi \xi^{-1} d \xi
$$


The use of Equation (1) and of the identifications $g(\xi)=\xi$ and $f(\xi)=\xi^{-1}$ yields

$$
\int_{0}^{x} \xi \xi^{-1} d \xi=\sum_{s=0}^{\infty}(-1)^{s} \frac{x^{s+2}}{(s+2) !}\left((-1)^{s} \frac{s !}{x^{s+1}}\right)=x \sum_{s=0}^{\infty} \frac{1}{(s+1)(s+2)}
$$

and being $\int_{0}^{x} 1 d \xi=x$, we deduce the identity

$$
\sum_{s=0}^{\infty} \frac{1}{(s+1)(s+2)}=1
$$

which is true, since its l.h.s., as shown below, reduces to the Telescopic series [6].

Example 2. The same procedure applied to $g(\xi)=\xi^{2}$ and $f(\xi)=\xi^{-1}$ provides the further identity [7]

$$
\sum_{s=0}^{\infty} \frac{1}{(s+1)(s+2)(s+3)}=\frac{1}{4}
$$

The extension of the procedure to the case $g(\xi)=\xi^{m}, f(\xi)=\xi^{-1}$ leads to the following series [7]

$$
\sum_{s=0}^{\infty} \frac{1}{(s+1)(s+2) \ldots(s+m+1)}=\sum_{s=0}^{\infty} \frac{s !}{(s+m+1) !}=\frac{1}{m ! m}
$$

which is a well known result (see, e.g., Formula 5.1.24.7 of ref. [8]).

Example 3. By using furthermore, in Equation (1), the identification $g(x)=x^{\alpha}$ and $f(x)=x^{-\alpha}$, $\forall \alpha \in\{\mathbb{R} \backslash \mathbb{Z}\}$, the inverse derivative Leibniz rule yields

$$
\sum_{s=0}^{\infty} \frac{(-1)^{s}}{\Gamma(s+\alpha+2) \Gamma(1-s-\alpha)}=\frac{1}{\Gamma(1+\alpha) \Gamma(1-\alpha)}
$$

which, after using the properties of the Gamma function " $\Gamma(\alpha) \Gamma(1-\alpha)=\frac{\pi}{\sin (\pi \alpha)}$ ", yields the telescopic sum

$$
\sum_{s=0}^{n} \frac{1}{(s+\alpha+1)} \frac{1}{(s+\alpha)}=\frac{1}{\alpha}
$$

The previous results are quite surprising, they are associated with generalized forms of telescopic series and can be embedded in an even wider context, as proved in the forthcoming section.

\section{Combinatorial Identities and Leibniz Formula}

We exploit the formalism outlined in the previous section to foresee a systematic procedure to obtain the identities of combinatorial type.

Example 4. We consider Equation (1) with $g(x)=1$ and $f(x)=x^{n}$, which yields

$$
\frac{x^{n+1}}{n+1}=\int x^{n} d x=\sum_{s=0}^{n}(-1)^{s} \frac{x^{s+1}}{(s+1) !} \frac{n !}{(n-s) !} x^{n-s}=x^{n+1} \sum_{s=0}^{n}\left(\begin{array}{l}
n \\
s
\end{array}\right) \frac{(-1)^{s}}{s+1}
$$

which implies

$$
\sum_{s=0}^{n}\left(\begin{array}{l}
n \\
s
\end{array}\right) \frac{(-1)^{s}}{s+1}=\frac{1}{n+1}
$$


Example 5. If we otherwise assume that $g(x)=x$ and $f(x)=x^{n}$, the following identity is straightforwardly obtained

$$
\sum_{s=0}^{n}\left(\begin{array}{l}
n \\
s
\end{array}\right) \frac{(-1)^{s}}{(s+1)(s+2)}=\frac{1}{n+2}
$$

indeed

$$
\begin{aligned}
& \frac{x^{n+2}}{n+2}=\hat{D}_{x}^{-1}\left(x \cdot x^{n}\right)=\sum_{s=0}^{n}(-1)^{s} \frac{x^{s+2}}{(s+2) !} \frac{n !}{(n-s) !} x^{n-s}=x^{n+2} \sum_{s=0}^{n}\left(\begin{array}{l}
n \\
s
\end{array}\right) \frac{(-1)^{s}}{(s+1)(s+2)} . \\
& \text { If we go on with } g(x)=x^{2} \text { and } f(x)=x^{n}, \text { we end up with } \\
& \frac{x^{n+3}}{n+3}=\sum_{s=0}^{n}(-1)^{s} 2 \frac{x^{s+3}}{(s+3) !} \frac{n !}{(n-s) !} x^{n-s}=2 x^{n+3} \sum_{s=0}^{n}\left(\begin{array}{l}
n \\
s
\end{array}\right) \frac{(-1)^{s}}{(s+1)(s+2)(s+3)}
\end{aligned}
$$

then

$$
\sum_{s=0}^{n}\left(\begin{array}{l}
n \\
s
\end{array}\right) \frac{(-1)^{s}}{(s+1)(s+2)(s+3)}=\frac{1}{2(n+3)}
$$

Remark 1. We can now manipulate Equations (15) and (16) to get a further result useful for the development of our discussion. It is evident that, by the use of the partial fractional expansion, we can write Equation (17) as

$$
\begin{aligned}
\frac{1}{n+2} & =\sum_{s=0}^{n}\left(\begin{array}{l}
n \\
s
\end{array}\right) \frac{(-1)^{s}}{(s+1)(s+2)}=\sum_{s=0}^{n}\left(\begin{array}{l}
n \\
s
\end{array}\right)(-1)^{s}\left(\frac{1}{s+1}-\frac{1}{s+2}\right) \\
& =\frac{1}{n+1}-\sum_{s=0}^{n}\left(\begin{array}{l}
n \\
s
\end{array}\right) \frac{(-1)^{s}}{s+2}
\end{aligned}
$$

which easily yields the identity

$$
\sum_{s=0}^{n}\left(\begin{array}{l}
n \\
s
\end{array}\right) \frac{(-1)^{s}}{s+2}=\frac{1}{(n+1)(n+2)}, \quad \forall n \in \mathbb{N} .
$$

The following expansion in terms of partial fractions

$$
\frac{2}{(s+1)(s+2)(s+3)}=\frac{1}{s+1}-\frac{2}{s+2}+\frac{1}{s+3}
$$

can be exploited to end up with

$$
\sum_{n=0}^{n}\left(\begin{array}{l}
n \\
s
\end{array}\right) \frac{(-1)^{s}}{s+3}=\frac{2}{(n+1)(n+2)(n+3)}
$$

Finally, by iterating the procedure, we end up with the proof of the following Theorem for two general formulae.

Theorem 1. $\forall n, m \in \mathbb{N}$

$$
\sum_{s=0}^{n}\left(\begin{array}{l}
n \\
s
\end{array}\right) \frac{(-1)^{s} s !}{(s+m+1) !}=\frac{1}{m !(n+m+1)} .
$$

and (see also Equation (52) later)

$$
\sum_{s=0}^{n}\left(\begin{array}{l}
n \\
s
\end{array}\right) \frac{(-1)^{s}}{s+m+1}=\frac{m !}{(n+1)(n+2) \ldots(n+m+1)}=\frac{n ! m !}{(n+m+1) !}
$$


For a deeper discussion on Theorem 1 and 2 (see later) see Refs. [9-11] where the results have already been derived in a different context.

The results of this section have indicated how methods of integro-differential nature are suitable to derive combinatorial identities and make progress in the Theory of Telescopic series. In the following sections, we will address these points in deeper detail and will show how further interesting results in these directions can be obtained.

\section{Inverse Derivatives and Generalized Telescopic Series}

A systematic investigation of the series quoted in Equation (11) has been undertaken in ref. [7]. Here, we adopt an analogous point of view and frame the results obtained so far within a different context. We use the following notation to indicate Telescopic series ( $T$ stands for telescopic).

\section{Example 6.}

$$
\begin{aligned}
& { }_{2} T_{2}:=\sum_{s=0}^{\infty} \frac{1}{(s+1)(s+2)}=\sum_{s=0}^{\infty}\left(\frac{1}{s+1}-\frac{1}{s+2}\right), \\
& { }_{3} T_{3}:=\sum_{s=0}^{\infty} \frac{1}{(s+1)(s+2)(s+3)}=\frac{1}{2} \sum_{s=0}^{\infty}\left(\frac{1}{s+1}-\frac{2}{s+2}+\frac{1}{s+3}\right),
\end{aligned}
$$

where we have used the convention

$$
\begin{aligned}
& { }_{m} T_{2}:=\sum_{s=0}^{\infty} \frac{1}{(s+m-1)(s+m)}, \quad m>1 \\
& { }_{m} T_{3}:=\sum_{s=0}^{\infty} \frac{1}{(s+m-2)(s+m-1)(s+m)}, \quad m>2 .
\end{aligned}
$$

Equation (27) can be eventually written as

$$
{ }_{3} T_{3}=\frac{1}{2}\left({ }_{2} T_{2}-{ }_{3} T_{2}\right)
$$

and being ${ }_{m} T_{2}=\frac{1}{(m-1)}$, we end up with

$$
\begin{aligned}
& { }_{2} T_{2}=1 \\
& { }_{3} T_{3}=\frac{1}{4} .
\end{aligned}
$$

Remark 2. The T notation allows us to write the identity in Equation (11) as

$$
{ }_{m+1} T_{m+1}=\frac{1}{m ! m} .
$$

Regarding the first of Equations (30), the relevant proof can also be achieved by the use of an alternative and more general procedure.

Remark 3. We use the Laplace transform method to write

$$
\sum_{s=0}^{n}\left(\begin{array}{l}
n \\
s
\end{array}\right) \frac{(-1)^{s}}{s+2}=\int_{0}^{\infty}\left(1-e^{-\sigma}\right)^{n} e^{-2 \sigma} d \sigma,
$$

on account of Equation (21), after summing over $n$, we end up with

$$
\sum_{n=0}^{\infty} \frac{1}{(n+1)(n+2)}=\int_{0}^{\infty} e^{-\sigma} d \sigma=1
$$


which is a restatement of Equation (9) on the basis of a totally different mean. The same procedure can be applied to get the proof for the other identities (see Section 5 for further comments).

Definition 2. $\forall n, m \in \mathbb{N}$, we introduce the notation (see Equation (25))

$$
{ }_{m} b_{n}:=\sum_{j=0}^{n}\left(\begin{array}{l}
n \\
j
\end{array}\right) \frac{(-1)^{j}}{j+m+1},
$$

to define the ${ }_{m} b_{n}$, which will be called Binomial Harmonic Numbers (BHN).

Remark 4. The iteration of the previous formalism yields (see also the forthcoming sections) the following identities. The BHN ${ }_{1} b_{n}$ and ${ }_{2} b_{n}$ have been given in Equations (21)-(23) and the generalized form $m b_{n}$ in Equation (25). The use of the integral representation yields

$$
{ }_{2} b_{n}=\int_{0}^{\infty}\left(1-e^{-\sigma}\right)^{n} e^{-3 \sigma} d \sigma
$$

which can be exploited to find

$$
\sum_{n=0}^{\infty}{ }_{2} b_{n}=\int_{0}^{\infty} e^{-2 \sigma} d \sigma=\frac{1}{2}
$$

and, more in general,

$$
\sum_{n=0}^{\infty}{ }_{m} b_{n}=\frac{1}{m},
$$

namely, the derivation of Equation (11) from a different perspective.

\section{Umbral Methods and Binomial Harmonic Numbers}

The harmonic numbers are defined (in refs. [12,13], we used a slightly different definition which here corresponds, strictly speaking, to the upper limit of the sum equal to $n-1$ ) as [14-17]

$$
h_{n}:=\sum_{s=0}^{n} \frac{1}{s+1}, \quad \forall n \in \mathbb{N},
$$

whose properties have been shown to be usefully studied using methods borrowed from umbral formalism. This point of view has been developed in a number of previous papers $[12,13,18,19]$ in which the associated algebraic procedures have been shown to be particularly effective. They have allowed the study of the relevant properties by straightforward means, paved the way to the introduction of generalized forms along with the tools to develop the underlying theoretical background. The methods suggested in [19] have given rise to a series of speculations and conjectures, and were then proved on a more solid basis in subsequent research [20-23].

In this section, we will use the umbral formalism to go deeper into the properties of the $B H N$. The use of the umbral notation adopted in $[3,18,19]$ allows us to manipulate complicated expressions and obtain remarkable results.

Definition 3. We impose that the umbral operator $\hat{a}$, acting on the vacuum $\varphi_{0}$, provides the position

$$
\hat{a}^{s} \varphi_{0}:=\frac{1}{s+1}, \quad \forall s \in \mathbb{N} .
$$

Property 1. The operator â satisfies the composition rule

$$
\hat{a}^{s} \hat{a}^{p}=\hat{a}^{s+p}, \quad \forall s, p \in \mathbb{N} .
$$

Then, we can state the following identity. 
Proposition 2. $\forall n \in \mathbb{N}$

$$
{ }_{0} b_{n}=(1-\hat{a})^{n} \varphi_{0}
$$

Proof. $\forall n \in \mathbb{N}$, by the use of the Newton binomial, of Definition 3 and of Property 1, we can write

$$
(1-\hat{a})^{n} \varphi_{0}=\sum_{s=0}^{n}\left(\begin{array}{l}
n \\
s
\end{array}\right)(-1)^{s} \hat{a}^{s} \varphi_{0}=\sum_{s=0}^{n}\left(\begin{array}{l}
n \\
s
\end{array}\right) \frac{(-1)^{s}}{s+1}={ }_{0} b_{n} .
$$

Corollary 1. The use of Equation (15) allows us to write

$$
(1-\hat{a})^{n} \varphi_{0}={ }_{0} b_{n}=\frac{1}{n+1} .
$$

Remark 5. We note that the umbral operator â acting on the same vacuum $\varphi_{0}$ yields the same algebraic result

$$
(1-\hat{a})^{n} \varphi_{0}=\hat{a}^{n} \varphi_{0}
$$

Indeed, for Proposition 2, Corollary 1 and Definition 3, we get

$$
(1-\hat{a})^{n} \varphi_{0}=\frac{1}{n+1}=\hat{a}^{n} \varphi_{0}
$$

Even though unenecessary, we note that Equation (43) does not imply that $(1-\hat{a})=\hat{a}$. It is not indeed an identity between operators but between algebraic quantities after the action of the umbral operators on the vacuum (as shown in [18], the differential realization of the umbral operator $\hat{a}$ is a shift operator $\hat{a}=e^{\partial_{z}}$, and the corresponding vacuum is $\varphi(z)=\frac{1}{z+1}$. The action on the vacuum of the operator $\hat{a}^{n}, \forall n \in \mathbb{N}$, is accordingly expressed through $\hat{a}^{n} \varphi_{0}=\left.e^{n \partial_{z}} \frac{1}{z+1}\right|_{z=0}=\frac{1}{n+1}$ and we also get

$$
(1-\hat{a})^{n} \varphi_{0}=\left.\left(1-e^{\partial_{z}}\right)^{n} \varphi(z)\right|_{z=0}=\left.\sum_{s=0}^{n}\left(\begin{array}{l}
n \\
s
\end{array}\right)(-1)^{s} \hat{a}^{s} \varphi(z)\right|_{z=0}=\sum_{s=0}^{n}\left(\begin{array}{l}
n \\
s
\end{array}\right) \frac{(-1)^{s}}{s+1}
$$

We can now clarify the remark after the identity (43), which in differenetial terms reads $\left.\left(1-e^{\partial_{z}}\right)^{n} \varphi(z)\right|_{z=0}=\left.e^{n \partial_{z}} \varphi(z)\right|_{z=0}$, which is true for $z=0$ but not in general; it is indeed easily checked that $\left.\left(1-e^{\partial_{z}}\right)^{n} \varphi(z) \neq e^{n \partial_{z}} \varphi(z)\right)$.

We iterate the method in the following way.

Proposition 3. $\forall n \in \mathbb{N}$

$$
\hat{a}(1-\hat{a})^{n} \varphi_{0}={ }_{1} b_{n}=\frac{1}{(n+1)(n+2)} .
$$

Proof. It is evident that

$$
(1-\hat{a})^{n+1} \varphi_{0}=\frac{1}{(n+1)+1} .
$$

We split the power so that

$$
(1-\hat{a})^{n+1} \varphi_{0}=(1-\hat{a})(1-\hat{a})^{n} \varphi_{0}=\left[(1-\hat{a})^{n}-\hat{a}(1-\hat{a})^{n}\right] \varphi_{0}
$$

and calculate the term $\hat{a}(1-\hat{a})^{n} \varphi_{0}$, by using Definition 3 and Property 1 , 


$$
\begin{aligned}
\hat{a}(1-\hat{a})^{n} \varphi_{0} & =\hat{a} \sum_{s=0}^{n}\left(\begin{array}{l}
n \\
s
\end{array}\right)(-1)^{s} \hat{a}^{s} \varphi_{0}=\sum_{s=0}^{n}\left(\begin{array}{l}
n \\
s
\end{array}\right)(-1)^{s} \hat{a}^{s+1} \varphi_{0} \\
& =\sum_{s=0}^{n}\left(\begin{array}{l}
n \\
s
\end{array}\right) \frac{(-1)^{s}}{(s+1)+1}={ }_{1} b_{n} .
\end{aligned}
$$

Furthermore, from Equations (45) and (46), we can establish the recurrence

$$
{ }_{0} b_{n}-{ }_{1} b_{n}=\frac{1}{n+2}
$$

which can be exploited to get the explicit form of ${ }_{1} b_{n}$, namely

$$
{ }_{1} b_{n}=\frac{1}{n+1}-\frac{1}{n+2}=\frac{1}{(n+1)(n+2)} .
$$

Corollary 2. The extension of the procedure yields, for ${ }_{2} b_{n}$, the recurrence

$$
{ }_{0} b_{n}-2{ }_{1} b_{n}+{ }_{2} b_{n}=\frac{1}{n+3}
$$

indeed

$$
\begin{aligned}
& \frac{1}{(n+2)+1}=(1-\hat{a})^{n+2} \varphi_{0}=(1-\hat{a})(1-\hat{a})^{n+1} \varphi_{0} \\
& =\left[(1-\hat{a})^{n+1}-\hat{a}(1-\hat{a})^{n+1}\right] \varphi_{0} \\
& =\left\{(1-\hat{a})^{n+1}-\hat{a}\left[(1-\hat{a})^{n}-\hat{a}(1-\hat{a})^{n}\right]\right\} \varphi_{0}={ }_{0} b_{n}-{ }_{1} b_{n}-{ }_{1} b_{n}+{ }_{2} b_{n}
\end{aligned}
$$

which, once solved for ${ }_{2} b_{n}$, yields

$$
{ }_{2} b_{n}=\frac{2}{(n+1)(n+2)(n+3)}
$$

which confirms the results in Equation (23). Equation (49) can be viewed as the umbral version of the partial fraction expansion exploited in the previous sections.

Theorem 2. All the BHN can be defined as binomial convolution of the lower order case $r b_{s}$

$$
{ }_{m} b_{n}=\sum_{s=0}^{n}\left(\begin{array}{l}
n \\
s
\end{array}\right)(-1)^{s} \sum_{r=0}^{m}\left(\begin{array}{c}
m \\
r
\end{array}\right)(-1)^{r}{ }_{r} b_{s}
$$

where

$$
{ }_{r} b_{s}=\hat{a}^{r}(1-\hat{a})^{s} \varphi_{0}=\frac{s ! r !}{(s+r+1) !} .
$$

Proof. The proof is obtained by the iteration of the previous procedure by noting that

$$
\begin{aligned}
{ }_{m} b_{n} & =\sum_{s=0}^{n}\left(\begin{array}{l}
n \\
s
\end{array}\right) \frac{(-1)^{s}}{s+m+1}=\sum_{s=0}^{n}\left(\begin{array}{l}
n \\
s
\end{array}\right)(-1)^{s}(1-\hat{a})^{m}(1-\hat{a})^{s} \varphi_{0} \\
& =\sum_{s=0}^{n}\left(\begin{array}{l}
n \\
s
\end{array}\right)(-1)^{s} \sum_{r=0}^{m}\left(\begin{array}{c}
m \\
r
\end{array}\right)(-1)^{r} \hat{a}^{r}(1-\hat{a})^{s} \varphi_{0} .
\end{aligned}
$$

As a further example, we consider the complementary forms of $B H N$. 
Definition 4. We introduce the Complementary Binomial Harmonic Number (CBHN) defined as

$$
m b_{n}^{+}:=\sum_{s=0}^{n}\left(\begin{array}{l}
n \\
s
\end{array}\right) \frac{1}{s+m+1}, \quad \forall m, n \in \mathbb{N} .
$$

Example 7. The CBHN can be constructed recursively from the identity

$$
{ }_{0} b_{n}^{+}=\frac{2^{n+1}-1}{n+1}
$$

easily proved by induction and further derived in Equation (74).

According to the same procedure as before, we set

$$
{ }_{0} b_{n}^{+}=(1+\hat{a})^{n} \varphi_{0}
$$

which yields

$$
\frac{2^{n+2}-1}{n+2}={ }_{0} b_{n+1}^{+}=(1+\hat{a})^{n+1} \varphi_{0}={ }_{0} b_{n}^{+}+{ }_{1} b_{n}^{+}
$$

and, once solved for ${ }_{1} b_{n}^{+}$, gets

$$
{ }_{1} b_{n}^{+}=\frac{2^{n+1} n+1}{(n+1)(n+2)} .
$$

The same method provides for ${ }_{2} b_{n}^{+}$

$$
\frac{2^{n+3}-1}{n+3}={ }_{0} b_{n+2}^{+}=(1+\hat{a})^{n+2} \varphi_{0}={ }_{0} b_{n}^{+}+2{ }_{1} b_{n}^{+}+{ }_{2} b_{n}^{+}
$$

thus finding

$$
\begin{gathered}
{ }_{2} b_{n}^{+}=\frac{2\left(2^{n}\left(n^{2}+n+2\right)-1\right)}{(n+1)(n+2)(n+3)}, \\
{ }_{3} b_{n}^{+}=\frac{2\left(2^{n} n\left(n^{2}+3 n+8\right)+3\right)}{(n+1)(n+2)(n+3)(n+4)}, \\
{ }_{4} b_{n}^{+}=\frac{2\left(2^{n}\left(n^{4}+6 n^{3}+23 n^{2}+18 n+24\right)-12\right)}{(n+1)(n+2)(n+3)(n+4)(n+5)}
\end{gathered}
$$

and by iteration, we can obtain other expressions for ${ }_{m} b_{n}^{+}$(with $m>4$ ), a synthetic expression in terms of ${ }_{2} F_{1}$ hypergeometric function can also be obtained but is no more informative than Equation (53), namely

$$
{ }_{m} b_{n}^{+}=\frac{{ }_{2} F_{1}(-n, m+1, m+2,-1)}{m+1}
$$

and the associated convolution finite sum

$$
\sum_{r=0}^{m}\left(\begin{array}{c}
m \\
r
\end{array}\right) r b_{n}^{+}=\sum_{r=0}^{m}\left(\begin{array}{l}
m \\
r
\end{array}\right) \sum_{s=0}^{n}\left(\begin{array}{l}
n \\
s
\end{array}\right) \frac{1}{s+r+1}=\frac{2^{n+m+1}-1}{n+m+1}
$$

In the previous section, we derived the identity shown in the forthcoming section which will be exploited to introduce an extension of the 2-order BHN.

Definition 5. We introduce the 2-order BHN

$$
{ }_{m} b_{n}^{(2)}:=\sum_{s=0}^{n}\left(\begin{array}{l}
n \\
s
\end{array}\right) \frac{(-1)^{s}}{2 s+m+1}, \quad \forall m, n \in \mathbb{N}
$$

Example 8. According to our umbral notation, the case $m=0$ can be written as 


$$
{ }_{0} b_{n}^{(2)}=\left(1-\hat{a}^{2}\right)^{n} \varphi_{0}
$$

which, according to the algebraic Properties 1 of $\hat{a}$ and the coefficients (52), allows us to write

$$
{ }_{0} b_{n}^{(2)}=(1+\hat{a})^{n}(1-\hat{a})^{n} \varphi_{0}=\sum_{s=0}^{n}\left(\begin{array}{l}
n \\
s
\end{array}\right) \hat{a}^{s}(1-\hat{a})^{n} \varphi_{0}=\sum_{s=0}^{n}\left(\begin{array}{l}
n \\
s
\end{array}\right){ }_{s} b_{n} .
$$

Along with Equation (52), the last identity yields the following further result

$$
{ }_{0} b_{n}^{(2)}=n !^{2} \sum_{s=0}^{n} \frac{1}{(n-s) !(n+s+1) !}=n !^{2} \frac{2^{2 n}}{(2 n+1) !}
$$

easily generalized to

$$
{ }_{m} b_{n}^{(2)}=\hat{a}^{m}\left(1-\hat{a}^{2}\right)^{n} \varphi_{0}=\sum_{s=0}^{n}\left(\begin{array}{l}
n \\
s
\end{array}\right) s+m b_{n}=n !^{2} \sum_{s=0}^{n} \frac{(s+m) !}{(n-s) ! s !(s+m+n+1) !} .
$$

Equations (67) and (68) are not satisfactory, in the sense that we are looking for a result not in the form of a series, albeit truncated.

The umbral technique can be used as a complementary procedure to frame the previous results within a different context.

\section{Combinatorial Identities, Integral Representations and Special Functions}

\subsection{Combinatorial Identities and Integral Representations}

The procedure we have just put forward has indicated that the formulae derived in Section 1 are a fairly straightforward means to getting old and new identities appearing in Combinatorics, Theory of Telescoping series and of the associated generalized forms. We have been indeed able to recover the results of ref. [7], as well as other disseminated in the mathematical literature, by following a unifying and straightforward procedure.

Before entering the discussion of a different topics, let us consider further examples.

Example 9. We start from the manipulation of the integral

$$
\begin{aligned}
\frac{(1+x)^{n+1}-1}{n+1} & =\int_{0}^{x}(1+\xi)^{n} d \xi=\left.\sum_{s=0}^{n}(-1)^{s} \frac{\xi^{s+1}}{(s+1) !} \frac{n !}{(n-s) !}(1+\xi)^{n-s}\right|_{0} ^{x} \\
& =\sum_{s=0}^{n}(-1)^{s} \frac{x^{s+1}}{s+1}\left(\begin{array}{l}
n \\
s
\end{array}\right)(1+x)^{n-s}
\end{aligned}
$$

which, after keeping e.g., $x=1$, yields

$$
\sum_{s=0}^{n}\left(\begin{array}{l}
n \\
s
\end{array}\right) \frac{(-1)^{s}}{s+1} 2^{n-s}=\frac{2^{n+1}-1}{n+1} .
$$

The identity in Equation (69) can be further handled and generalized along the same lines we discussed in the previous sections, thus getting, for example,

$$
\sum_{s=0}^{n}(-1)^{s} \frac{x^{s+2}}{(s+1)(s+2)}\left(\begin{array}{l}
n \\
s
\end{array}\right)(1+x)^{n-s}=\frac{(n x+x-1)(1+x)^{n+1}+1}{(n+1)(n+2)} .
$$

After breaking the l.h.s. in partial sums, we also find for $x=1$,

$$
\sum_{s=0}^{n}\left(\begin{array}{l}
n \\
s
\end{array}\right) \frac{(-1)^{s}}{(s+2)} 2^{n-s}=\frac{2^{n+2}-(n+3)}{(n+1)(n+2)} .
$$

Example 10. The slightly generalized form of Equation (69) 


$$
\frac{(a+b x)^{n+1}-a^{n+1}}{b(n+1)}=\int_{0}^{x}(a+b \xi)^{n} d \xi=\sum_{s=0}^{n}(-1)^{s} \frac{x^{s+1}}{(s+1) !} \frac{n !}{(n-s) !}(a+b x)^{n-s} b^{s}
$$

can be exploited to derive further identities. By keeping, for example, $a=2, b=1, x=-1$, we find

$$
\sum_{s=0}^{n}\left(\begin{array}{l}
n \\
s
\end{array}\right) \frac{1}{s+1}=\frac{2^{n+1}-1}{n+1} .
$$

Example 11. A further identity of pivotal importance, for the present purposes, is provided by

$$
\begin{aligned}
\sum_{s=0}^{n}\left(\begin{array}{l}
n \\
s
\end{array}\right) \frac{(-1)^{s}}{2 s+1} & =\int_{0}^{1}\left(1-\xi^{2}\right)^{n} d \xi=\int_{0}^{1}\left(1-\xi^{2}\right)^{n} d \xi=\frac{1}{2} B\left(\frac{1}{2}, n+1\right) \\
& =\left(\frac{3}{2}+n\right) B\left(\frac{3}{2}, n+1\right)
\end{aligned}
$$

where $B(x, y)$ being the Euler Beta function and, by expoliting the B-function properties, we obtain

$$
\sum_{s=0}^{n}\left(\begin{array}{l}
n \\
s
\end{array}\right) \frac{(-1)^{s}}{2 s+1}=\frac{2^{2 n} n !^{2}}{(2 n+1) !}
$$

indeed

$$
\frac{1}{2} B\left(\frac{1}{2}, n+1\right)=\frac{1}{2} \frac{\Gamma\left(\frac{1}{2}\right) \Gamma(n+1)}{\Gamma\left(n+\frac{3}{2}\right)}=\frac{1}{2} \sqrt{\pi} n ! \frac{4^{n+1}(n+1) !}{(2(n+1) !) \sqrt{\pi}}=\frac{2^{2 n} n !^{2}}{(2 n+1) !} .
$$

Furthermore, by setting $x=1$ and using the transformation $\xi^{2}=t$, the result in Equation (75) is achieved. In more general terms, we also get

$$
\sum_{s=0}^{n}\left(\begin{array}{l}
n \\
s
\end{array}\right) \frac{(-1)^{s}}{\alpha s+\beta}=\int_{0}^{1}\left(1-\xi^{\alpha}\right)^{n} \xi^{\beta-1} d \xi=\frac{1}{\alpha} B\left(\frac{\beta}{\alpha}, n+1\right) .
$$

Example 12. Furthermore, by using the Laplace transform method, we get the integral representation of the two equivalent series

$$
\begin{aligned}
& \sum_{s=0}^{n}(-1)^{s} \frac{x^{s}}{(s+2)}\left(\begin{array}{l}
n \\
s
\end{array}\right)(1+x)^{n-s}=\sum_{s=0}^{n}\left(\begin{array}{l}
n \\
s
\end{array}\right) \frac{x^{s}}{(s+1)(s+2)}=\phi_{n}(x), \\
& \phi_{n}(x)=\int_{0}^{\infty}\left[1+\left(1-e^{-\sigma}\right) x\right]^{n} e^{-2 \sigma} d \sigma
\end{aligned}
$$

whose sum is

$$
\begin{aligned}
\sum_{s=0}^{n}(-1)^{s} \frac{x^{s}}{(s+2)}\left(\begin{array}{l}
n \\
s
\end{array}\right)(1+x)^{n-s} & =\sum_{s=0}^{n}\left(\begin{array}{l}
n \\
s
\end{array}\right) \frac{x^{s}}{(s+1)(s+2)} \\
& =\frac{(x+1)^{n+2}-(n x+2 x+1)}{x^{2}(n+1)(n+2)} .
\end{aligned}
$$

The integral representation can also be exploited to show that the sum $\sum_{n=0}^{\infty} \phi_{n}(x)$ is actually converging for $x<0$ et $0<|x|<1$. 
Example 13. The umbral method developed in the section can be further exploited by noting that the previous procedure allows the derivation of the identities

$$
\left(1-\hat{a}^{2}\right)^{n} \varphi_{0}={ }_{0} b_{n}^{(2)}=n ! \frac{\Gamma\left(\frac{3}{2}\right)}{\Gamma\left(n+\frac{3}{2}\right)} .
$$

We can take advantage from them by applying the paradigm outlined in the introductory section. Accordingly, we find

$$
\left(1-\hat{a}^{2}\right)^{n+1} \varphi_{0}=(n+1) ! \frac{\Gamma\left(\frac{3}{2}\right)}{\Gamma\left(n+\frac{5}{2}\right)} .
$$

By following the technique of Equation (46) with Equation (52), we note that

$$
\left(1-\hat{a}^{2}\right)^{n+1} \varphi_{0}=\left(1-\hat{a}^{2}\right)\left(1-\hat{a}^{2}\right)^{n} \varphi_{0}={ }_{0} b_{n}^{(2)}-{ }_{2} b_{n}^{(2)},
$$

then we can obtain the identity

$$
{ }_{2} b_{n}^{(2)}=\sum_{s=0}^{n}\left(\begin{array}{l}
n \\
s
\end{array}\right) \frac{(-1)^{s}}{2 s+3}=n ! \Gamma\left(\frac{3}{2}\right) \frac{\Gamma\left(n+\frac{5}{2}\right)-(n+1) \Gamma\left(n+\frac{3}{2}\right)}{\Gamma\left(n+\frac{3}{2}\right) \Gamma\left(n+\frac{5}{2}\right)}=\frac{n !}{2} \frac{\Gamma\left(\frac{3}{2}\right)}{\Gamma\left(n+\frac{5}{2}\right)} .
$$

Example 14. We can generalize the method to any real power

$$
m b_{n}^{(k)}=\sum_{s=0}^{n}\left(\begin{array}{l}
n \\
s
\end{array}\right) \frac{(-1)^{s}}{k s+m+1}=\frac{n !}{k} \frac{\Gamma\left(\frac{m+1}{k}\right)}{\Gamma\left(\frac{k n+m+k+1}{k}\right)}, \quad \forall k \in \mathbb{R} .
$$

\subsection{Leibniz Rule, Umbral Methods and Special Functions}

By considering now the two variable Hermite polynomial $H_{n}(x, y)$ [5]

$$
H_{n}(x, y)=n ! \sum_{r=0}^{\left\lfloor\frac{n}{2}\right\rfloor} \frac{x^{n-2 r} y^{r}}{(n-2 r) ! r !}, \quad \forall x, y \in \mathbb{R}, \forall n \in \mathbb{N},
$$

we can provide the following example.

Example 15. By reminding the Hermite polynomials property

$$
\partial_{x}^{s} H_{n}(x, y)=\frac{n !}{(n-s) !} H_{n-s}(x, y)
$$

It is worth considering the integral

$$
\begin{aligned}
\frac{H_{n+1}(x, y)-H_{n+1}(0, y)}{n+1} & =\int_{0}^{x} H_{n}(\xi, y) d \xi=\sum_{s=0}^{n}(-1)^{s} \frac{x^{s+1}}{(s+1) !} \frac{n !}{(n-s) !} H_{n-s}(x, y) \\
& =\sum_{s=0}^{n}(-1)^{s}\left(\begin{array}{l}
n \\
s
\end{array}\right) \frac{x^{s+1}}{(s+1)} H_{n-s}(x, y)
\end{aligned}
$$

which is by no means surprising since the Hermite polynomials satisfy a monic type derivative [24].

Furthermore, the use of the umbral notation $[18,25]$ 


$$
\begin{aligned}
& H_{n}(x, y)=(x+y \hat{h})^{n} \theta_{0}, \quad \forall x, y \in \mathbb{R}, \forall n \in \mathbb{N}, \\
& y \hat{h}^{r} \theta_{0}:=\theta_{r}=\frac{y^{\frac{r}{2}} r !}{\Gamma\left(\frac{r}{2}+1\right)}\left|\cos \left(r \frac{\pi}{2}\right)\right|=\left\{\begin{array}{ll}
0 & (2 s) ! \\
y^{s} \frac{(2 s+1}{s !} & r=2 s
\end{array} \quad \forall s \in \mathbb{Z} .\right.
\end{aligned}
$$

allows us to writes

$$
\sum_{s=0}^{n}(-1)^{s} \frac{x^{s+1}}{(s+1) !} \frac{n !}{(n-s) !} H_{n-s}(x, y)=x \sum_{s=0}^{n}(-1)^{s}\left(\begin{array}{l}
n \\
s
\end{array}\right) \frac{x^{s}}{s+1}(x+y \hat{h})^{n-s} \theta_{0}
$$

and also the identities

$$
\begin{aligned}
x \sum_{s=0}^{n}(-1)^{s}\left(\begin{array}{l}
n \\
s
\end{array}\right) \frac{x^{s}}{s+1}(x+y \hat{h})^{n-s} \theta_{0} & =x \int_{0}^{\infty}\left[x\left(1-e^{-\sigma}\right)+y \hat{h}\right]^{n} e^{-\sigma} d \sigma \\
& =x \int_{0}^{\infty} H_{n}\left(x\left(1-e^{-\sigma}\right), y\right) e^{-\sigma} d \sigma
\end{aligned}
$$

which lead to the unexpected integral representation

$$
\int_{0}^{\infty} H_{n}\left(x\left(1-e^{-\sigma}\right), y\right) e^{-\sigma} d \sigma=\frac{H_{n+1}(x, y)-H_{n+1}(0, y)}{(n+1) x} .
$$

The identities we have discussed so far are just a few examples of the possibilities offered by the negative derivative formalism. A forthcoming investigation will provide a more carefully discussion in this respect.

In the final section, we will show how a formalism of umbral nature can be exploited to provide a useful complement for the treatment outlined in the previous sections.

\section{Final Comments}

This paper has been aimed at developing a self-contained treatment of the Theory of Combinatorial identities and of generalized harmonic numbers by embedding them within the context of a twofold complementary formalism.

The results we have obtained have such wide implications and cannot be comprised in the space of an article. We would like, however, to note that the negative derivative formalism is essentially a reformulation of the Leibniz rule. The ordinary formula is also a very useful tool to derive combinatorial identities.

Example 16. We note that, since $\partial_{x}^{n} x^{3 n}=\frac{(3 n) !}{(2 n) !} x^{2 n}$, comparing with $\partial_{x}^{n}\left(x^{n} x^{2 n}\right)$ and after using the Leibniz rule, we get

$$
\sum_{s=0}^{n}\left(\begin{array}{l}
n \\
s
\end{array}\right)\left(\begin{array}{c}
2 n \\
s
\end{array}\right)=\frac{(3 n) !}{(2 n) ! n !}
$$

which is easily generalized to (a particular case of the Chu-Vandermonde identity)

$$
\sum_{s=0}^{n}\left(\begin{array}{c}
k n \\
s
\end{array}\right)\left(\begin{array}{l}
n \\
s
\end{array}\right)=\frac{((k+1) n) !}{(k n) ! n !}=\left(\begin{array}{c}
(k+1) n \\
n
\end{array}\right) \text {. }
$$

The umbral formalism deserves further comments.

Definition 6. We introduce Binomial Convoluted Harmonic Number (BCHN) as 


$$
{ }_{m} \beta_{n}:=\sum_{s=0}^{n}\left(\begin{array}{l}
n \\
s
\end{array}\right)(-1)^{s} h_{m+s}, \quad \forall m, n \in \mathbb{N},
$$

where $h_{m+s}$ are the harmonic numbers cited in Equation (38).

The relevant properties can be studied by the use of the methods outlined in Section 4 .

Example 17. According to the umbral notation in $[12,13], \hat{h}^{r} \psi_{0}:=h_{r}$, we can set

$$
{ }_{m} \beta_{n}:=\hat{h}^{m}(1-\hat{h})^{n} \psi_{0}
$$

by the way

$$
\begin{aligned}
\hat{h}^{m}(1-\hat{h})^{n} \psi_{0} & =\hat{h}^{m} \sum_{r=0}^{n}\left(\begin{array}{l}
n \\
r
\end{array}\right)(-1)^{r} \hat{h}^{r} \psi_{0}=\sum_{r=0}^{n}\left(\begin{array}{l}
n \\
r
\end{array}\right)(-1)^{r} \hat{h}^{m+r} \psi_{0} \\
& =\sum_{r=0}^{n}\left(\begin{array}{l}
n \\
r
\end{array}\right)(-1)^{r} h_{m+r}={ }_{m} \beta_{n} .
\end{aligned}
$$

We prove that

$$
{ }_{0} \beta_{n}=-\frac{1}{n(n+1)}
$$

indeed

$$
\begin{aligned}
{ }_{0} \beta_{n} & =\sum_{s=0}^{n}\left(\begin{array}{l}
n \\
s
\end{array}\right)(-1)^{s} \sum_{r=0}^{s} \frac{1}{r+1}=\sum_{s=0}^{n}\left(\begin{array}{l}
n \\
s
\end{array}\right)(-1)^{s} \sum_{r=0}^{s} \int_{0}^{1} x^{r} d x \\
& =\sum_{s=0}^{n}\left(\begin{array}{l}
n \\
s
\end{array}\right)(-1)^{s} \int_{0}^{1} \frac{x^{s+1}-1}{x-1} d x \\
& =-\int_{0}^{1} \frac{x(1-x)^{n}}{x-1} d x=-\frac{1}{n(n+1)}
\end{aligned}
$$

and it is evident that, once we know $\beta_{n}$, we can infer recursively all the ${ }_{m} \beta_{n}$.

$$
{ }_{0} \beta_{n+1}=-\frac{1}{(n+1)(n+2)},
$$

from Equation (96), we find

$$
{ }_{0} \beta_{n+1}=(1-\hat{h})(1-\hat{h})^{n} \psi_{0}
$$

which yields the recurrence

$$
{ }_{0} \beta_{n+1}={ }_{0} \beta_{n}-{ }_{1} \beta_{n}
$$

and which allows the computation of the BCHN for $m=1$

$$
{ }_{1} \beta_{n}=-\frac{2}{n(n+1)(n+2)} .
$$

The extension of the procedure yields, for $m=2$,

$$
{ }_{2} \beta_{n}=-\frac{6}{n(n+1)(n+2)(n+3)}
$$

which eventually suggests that

$$
{ }_{m} \beta_{n}=-\frac{(m+1) !}{n(n+1)(n+2) \ldots(n+m+1)}=-\frac{(n-1) !(m+1) !}{(n+m+1) !} .
$$


This last comment completes the paper, and the results of which are summarized in the following Tables 1-3.

The article has addressed a number of points either in calculus or the Number Theory. We believe that its most noticeable achievement is having established a clear link between the formalism of negative derivatives and the properties of harmonic numbers. Albeit some of the results we have discussed have been obtained in previous authoritative papers, our efforts have been directed towards opening an alternative research avenue, eventually connecting apparently separated fields. In a forthcoming publication, we will extend the methodology to fractional integration and to the many variable cases.

Table 1. Combinatorial Identities.

\begin{tabular}{|c|c|c|}
\hline Binomial Harmonic Numbers & Umbral Images & Complementary BHN \\
\hline$m b_{n}=\sum_{s=0}^{n}\left(\begin{array}{l}n \\
s\end{array}\right) \frac{(-1)^{s}}{s+m+1}$ & $\hat{a}^{n} \varphi_{0}=\frac{1}{n+1}$ & $m b_{n}^{+}=\sum_{s=0}^{n}\left(\begin{array}{l}n \\
s\end{array}\right) \frac{1}{s+m+1}$ \\
\hline${ }_{0} b_{n}=\sum_{s=0}^{n}\left(\begin{array}{l}n \\
s\end{array}\right) \frac{(-1)^{s}}{s+1}=\frac{1}{n+1}$ & $(1 \mp \hat{a})^{n} \varphi_{0}$ & ${ }_{0} b_{n}^{+}=\frac{2^{n+1}-1}{n+1}$ \\
\hline${ }_{1} b_{n}=\sum_{s=0}^{n}\left(\begin{array}{l}n \\
s\end{array}\right) \frac{(-1)^{s}}{s+2}=\frac{1}{(n+1)(n+2)}$ & $\hat{a}(1 \mp \hat{a})^{n} \varphi_{0}$ & ${ }_{1} b_{n}^{+}=\frac{2^{n+1} n+1}{(n+1)(n+2)}$ \\
\hline $\begin{aligned}{ }_{2} b_{n}= & \sum_{s=0}^{n}\left(\begin{array}{l}n \\
s\end{array}\right) \frac{(-1)^{s}}{s+3} \\
& =\frac{2}{(n+1)(n+2)(n+3)}\end{aligned}$ & $\hat{a}^{2}(1 \mp \hat{a})^{n} \varphi_{0}$ & ${ }_{2} b_{n}^{+}=\frac{2\left(2^{n}\left(n^{2}+n+2\right)-1\right)}{(n+1)(n+2)(n+3)}$ \\
\hline$\ldots$ & $\ldots$ & $\ldots$ \\
\hline$m b_{n}=\frac{n ! m !}{(n+m+1) !}$ & $\hat{a}^{m}(1 \mp \hat{a})^{n} \varphi_{0}$ & $m b_{n}^{+}=\frac{{ }_{2} F_{1}(-n, m+1, m+2,-1)}{m+1}$ \\
\hline High Order BHN & U.I. & Recursivity \\
\hline$m b_{n}^{(k)}=\sum_{s=0}^{n}\left(\begin{array}{l}n \\
s\end{array}\right) \frac{(-1)^{s}}{k s+m+1}$ & & \\
\hline${ }_{0} b_{n}^{(2)}=\sum_{s=0}^{n}\left(\begin{array}{l}n \\
s\end{array}\right) \frac{(-1)^{s}}{2 s+1}=\frac{n ! \Gamma\left(\frac{3}{2}\right)}{\Gamma\left(n+\frac{3}{2}\right)}$ & $\left(1-\hat{a}^{2}\right)^{n} \varphi_{0}$ & $=\sum_{s=0}^{n}\left(\begin{array}{l}n \\
s\end{array}\right)_{s} b_{n} \Rightarrow \sum_{s=0}^{n} \frac{1}{(n-s) !(s+n+1) !}=\frac{2^{2 n}}{(2 n+1) !}$ \\
\hline${ }_{1} b_{n}^{(2)}=\sum_{s=0}^{n}\left(\begin{array}{l}n \\
s\end{array}\right) \frac{(-1)^{s}}{2 s+2}=\frac{1}{2(n+1)}$ & $\hat{a}\left(1-\hat{a}^{2}\right)^{n} \varphi_{0}$ & $=\sum_{s=0}^{n}\left(\begin{array}{l}n \\
s\end{array}\right)_{s+1} b_{n} \Rightarrow \sum_{s=0}^{n} \frac{(s+1)}{(n-s) !(s+n+2) !}=\frac{1}{2 n !(n+1) !}$ \\
\hline${ }_{2} b_{n}^{(2)}=\sum_{s=0}^{n}\left(\begin{array}{l}n \\
s\end{array}\right) \frac{(-1)^{s}}{2 s+3}=\frac{n ! \Gamma\left(\frac{3}{2}\right)}{2 \Gamma\left(n+\frac{5}{2}\right)}$ & $\hat{a}^{2}\left(1-\hat{a}^{2}\right)^{n} \varphi_{0}$ & $=\sum_{s=0}^{n}\left(\begin{array}{l}n \\
s\end{array}\right)_{s+2} b_{n} \Rightarrow \begin{array}{l}\sum_{s=0}^{n} \frac{(s+1)(s+2)}{(n-s) !(s+n+3) !} \\
=\frac{2^{2 n}}{(2 n+3)(2 n+1) !}\end{array}$ \\
\hline$\ldots$ & $\ldots$ & $\ldots$ \\
\hline 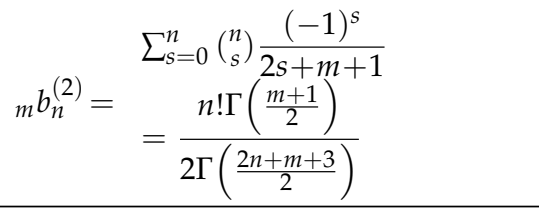 & $\hat{a}^{m}\left(1-\hat{a}^{2}\right)^{n} \varphi_{0}$ & $=\sum_{s=0}^{n}\left(\begin{array}{l}n \\
s\end{array}\right)_{s+m} b_{n} \Rightarrow \begin{array}{l}\sum_{s=0}^{n} \frac{(s+m) !}{(n-s) ! s !(s+m+n+1) !} \\
=\frac{\Gamma\left(\frac{m+1}{2}\right)}{2 n ! \Gamma\left(\frac{2 n+m+3}{2}\right)}\end{array}$ \\
\hline$\ldots$ & $\ldots$ & $\ldots$ \\
\hline$m b_{n}^{(k)}=\frac{n ! \Gamma\left(\frac{m+1}{k}\right)}{k \Gamma\left(\frac{k n+m+k+1}{k}\right)}$ & $\hat{a}^{m}\left(1-\hat{a}^{k}\right)^{n} \varphi_{0}$ & $\begin{aligned}=\sum_{s=0}^{n}\left(\begin{array}{l}n \\
s\end{array}\right) \frac{k}{2} s+m & b_{n}^{\left(\frac{k}{2}\right)} \Rightarrow \begin{array}{l}\sum_{s=0}^{n} \frac{\Gamma\left(s+2 \frac{m+1}{k}\right)}{(n-s) ! s ! \frac{k}{2} \Gamma\left(n+s+1+2 \frac{m+1}{k}\right)} \\
=\frac{\Gamma\left(\frac{m+1}{k}\right)}{k n ! \Gamma\left(\frac{k n+m+k+1}{k}\right)}\end{array}\end{aligned}$ \\
\hline
\end{tabular}


Table 2. Combinatorial Identities.

\begin{tabular}{|c|c|c|}
\hline BHN Combinatorics & Integral Representations & Sum \\
\hline$\frac{1}{n+1}={ }_{0} b_{n}$ & ${ }_{0} b_{n}=\int_{0}^{\infty}\left(1-e^{-\sigma}\right)^{n} e^{-\sigma} d \sigma=\frac{1}{n+1}$ & $\sum_{n=0}^{\infty} b_{n}=\infty$ \\
\hline$\frac{1}{n+2}={ }_{0} b_{n}-{ }_{1} b_{n}$ & ${ }_{1} b_{n}=\begin{array}{l}\int_{0}^{\infty}\left(1-e^{-\sigma}\right)^{n} e^{-2 \sigma} d \sigma \\
=\frac{1}{(n+1)(n+2)}\end{array}$ & $\sum_{n=0}^{\infty}{ }_{1} b_{n}=1$ \\
\hline$\frac{1}{n+3}={ }_{0} b_{n}-2{ }_{1} b_{n}+{ }_{2} b_{n}$ & ${ }_{2} b_{n}=\begin{array}{l}\int_{0}^{\infty}\left(1-e^{-\sigma}\right)^{n} e^{-3 \sigma} d \sigma \\
=\frac{2}{(n+1)(n+2)(n+3)}\end{array}$ & $\sum_{n=0}^{\infty} b_{n}=\frac{1}{2}$ \\
\hline$\ldots$ & $\ldots$ & $\ldots$ \\
\hline$\frac{1}{n+m+1}=\sum_{r=0}^{m}\left(\begin{array}{c}m \\
r\end{array}\right)(-1)^{r}{ }_{r} b_{n}$ & 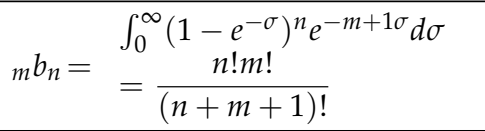 & $\sum_{n=0}^{\infty} m b_{n}=\frac{1}{m}$ \\
\hline \multirow[t]{2}{*}{ Binomial TS } & Integral Representations & Telescopic Series \\
\hline & BTS; $\quad$ TS & ${ }_{m} T_{k}=\sum_{s=0}^{\infty} \frac{(s+m-k) !}{(s+m) !}$ \\
\hline$\sum_{s=0}^{n}\left(\begin{array}{l}n \\
s\end{array}\right) \frac{(-1)^{s}}{s+1}=\frac{1}{n+1}$ & $\int x^{n} d x$ & $\cdots$ \\
\hline$\sum_{s=0}^{n}\left(\begin{array}{l}n \\
s\end{array}\right) \frac{(-1)^{s}}{(s+1)(s+2)}=\frac{1}{n+2}$ & $\int x^{n+1} d x ; \int_{0}^{x} \xi \xi^{-1} d \xi$ & ${ }_{2} T_{2}=\sum_{s=0}^{\infty} \frac{1}{(s+1)(s+2)}=1$ \\
\hline$\sum_{s=0}^{n}\left(\begin{array}{l}n \\
s\end{array}\right) \frac{(-1)^{s}}{(s+1)(s+2)(s+3)}=\frac{1}{2(n+3)}$ & $\int x^{n+2} d x ; \int_{0}^{x} \xi^{2} \xi^{-1} d \xi$ & ${ }_{3} T_{3}=\sum_{s=0}^{\infty} \frac{1}{(s+1)(s+2)(s+3)}=\frac{1}{4}$ \\
\hline$\ldots$ & $\ldots$ & $\ldots$ \\
\hline$\sum_{s=0}^{n}\left(\begin{array}{l}n \\
s\end{array}\right) \frac{(-1)^{s} s !}{(s+m+1) !}=\frac{1}{m !(m+n+1)}$ & $\int x^{n+m} d x ; \quad \int_{0}^{x} \xi^{m} \xi^{-1} d \xi$ & ${ }_{m+1} T_{m+1}=\sum_{s=0}^{\infty} \frac{s !}{(s+m+1) !}=\frac{1}{m ! m}$ \\
\hline
\end{tabular}

Table 3. Combinatorial Identities.

\begin{tabular}{ll}
\hline Integral Representations & Further Results \\
\hline$\left.\int_{0}^{x}(1+\xi)^{n} d \xi \Rightarrow\right|_{x=1}$ & $\sum_{s=0}^{n}\left(\begin{array}{l}n \\
s\end{array}\right) \frac{(-1)^{s} 2^{n-s}}{s+1}=\frac{2^{n+1}-1}{n+1}$ \\
\hline$\left.\int_{0}^{x}(1+\xi)^{n} \xi d \xi \Rightarrow\right|_{x=1}$ & $\sum_{s=0}^{n}\left(\begin{array}{l}n \\
s\end{array}\right) \frac{(-1)^{s} 2^{n-s}}{s+2}=\frac{2^{n+2}-(n+3)}{(n+1)(n+2)}$ \\
\hline$\left.\int_{0}^{x}(a+b \xi)^{n} d \xi \Rightarrow\right|_{x=-1, a=2, b=1}$ & $\sum_{s=0}^{n}\left(\begin{array}{l}n \\
s\end{array}\right) \frac{1}{s+1}=\frac{2^{n+1}-1}{n+1}$ \\
\hline $\int_{0}^{1}\left(1-\xi^{2}\right)^{n} d \xi \Rightarrow$ & $\sum_{s=0}^{n}\left(\begin{array}{l}n \\
s\end{array}\right) \frac{(-1)^{s}}{2 s+1}=\frac{2^{2 n} n !^{2}}{(2 n+1) !}=\frac{1}{2} B\left(\frac{1}{2}, n+1\right)=\left(\frac{3}{2}+n\right) B\left(\frac{3}{2}, n+1\right)$ \\
\hline $\int_{0}^{1}\left(1-\xi^{\alpha}\right)^{n} \xi^{\beta-1} d \xi \Rightarrow$ & $\sum_{s=0}^{n}\left(\begin{array}{l}n \\
s\end{array}\right) \frac{(-1)^{s}}{\alpha s+\beta}=\frac{1}{\alpha} B\left(\frac{\beta}{\alpha}, n+1\right)$ \\
\hline $\int_{0}^{x} H_{n}(\xi, y) d \xi \Rightarrow$ & $\sum_{s=0}^{n}\left(\begin{array}{l}n \\
s\end{array}\right) \frac{(-1)^{s} x^{s+1}}{s+1} H_{n-s}(x, y)=\frac{H_{n+1}(x, y)-H_{n+1}(0, y)}{n+1}$ \\
\hline
\end{tabular}


Table 3. Cont.

\begin{tabular}{ll}
\hline Binomial Convoluted HN & Umbral Image \\
\hline${ }_{m} \beta_{n}=\sum_{s=0}^{n}\left(\begin{array}{c}n \\
s\end{array}\right)(-1)^{s} h_{m+s}$ & $\hat{h}^{r} \psi_{0}=h_{r}=\sum_{s=0}^{r} \frac{1}{s+1}$ \\
\hline${ }_{0} \beta_{n}=-\frac{1}{n(n+1)}$ & $(1-\hat{h})^{n} \psi_{0}$ \\
\hline${ }_{1} \beta_{n}=-\frac{2}{n(n+1)(n+2)}$ & $\hat{h}(1-\hat{h})^{n} \psi_{0}$ \\
\hline${ }_{2} \beta_{n}=-\frac{6}{n(n+1)(n+2)(n+3)}$ & $\hat{h}^{2}(1-\hat{h})^{n} \psi_{0}$ \\
\hline$\ldots$ & $\ldots$ \\
\hline${ }_{m} \beta_{n}=-\frac{(n-1) !(m+1) !}{(n+m+1) !}$ & $\hat{h}^{m}(1-\hat{h})^{n} \psi_{0}$ \\
\hline
\end{tabular}

Author Contributions: Conceptualization: G.D.; methodology: G.D., S.L.; data curation: S.L.; validation: G.D., S.L., R.M.P.; formal analysis: G.D., S.L.; writing-original draft preparation: G.D.; writing-review and editing: S.L. All authors have read and agreed to the published version of the manuscript.

Funding: This research received no external funding.

Acknowledgments: The work of S. Licciardi was supported by an Enea Research Center individual fellowship. R.M. Pidatella thanks the University of Catania, project "Piano della Ricerca 2016/2018 Linea di intervento 2", for the partial support of this work. The co-authors thank R.M. Pidatella for the hospitality received at the University of Catania as part of the project "Piano della Ricerca 2016/2018 Linea di intervento 2".

Conflicts of Interest: The authors declare no conflict of interest.

\section{References}

1. Dattoli, G.; Germano, B.; Martinelli, M.R.; Ricci, P.E. Negative derivatives and special functions. Appl. Math. Comp. 2010, 217, 3924-3928. [CrossRef]

2. Dattoli, G.; Germano, B.; Martinelli, M.R.; Ricci, P.E. The negative derivative operator. Integral Transform. Spec. Funct. 2008, 19, 259-266. [CrossRef]

3. Babusci, D.; Dattoli, G.; Licciardi, S.; Sabia, E. Mathematical Methods for Physics; World Scientific: Singapore, 2019.

4. Oldham, K.B.; Spanier, J. The Fractional Calculus: Theory and Applications of Differentiation and Integration to Arbitrary Order. Math. Sci. Eng. 1974, 111, 240.

5. Dattoli, G.; Licciardi, S. Operational, Umbral Methods, Borel Transform and Negative Derivative Operator Techniques. Integral Transform. Spec. Funct. 2020, 31, 192-220. [CrossRef]

6. Kac, V.G.; Cheung, P. Quantum Calculus; Universitext; Springer: New York, NY, USA, 2002.

7. Ash, J.M.; Catoiu, S. Telescoping, rational-valued series, and zeta functions. Trans. Amer. Math. Soc. 2005, 357, 3339-3358. [CrossRef]

8. Prudnikov, A.P.; Brychkov, Y.A.; Marichev, O.I. Integrals and Series; Gordon and Breach: Glasgow, UK, 1986 ; Volume 1.

9. Batir, N.; Chen, K.W. Finite Hurwitz-Lerch functions. Filomat 2019, 33, 101-109. [CrossRef]

10. Chu, W. Summation formulae involving harmonic numbers. Filomat 2012, 26, 143-152. [CrossRef]

11. Chu, W.; Yan, Q.L. Combinatorial identities on binomial coefficients and harmonic numbers. Util. Math. 2008, 75, 51-66.

12. Dattoli, G.; Germano, B.; Licciardi, S.; Martinelli, M.R.: Umbral Methods and Harmonic Numbers. Axioms 2018, 7, 62.

13. Dattoli, G.; Licciardi, S.; Sabia, E.; Srivastava, H.M. Some Properties and Generating Functions of Generalized Harmonic Numbers. Mathematics 2019, 7, 577. [CrossRef]

14. Dattoli, G.; Srivastava, H.M. A Note on Harmonic Numbers, Umbral Calculus and Generating Functions. Appl. Math. Lett. 2008, 21, 686-693. [CrossRef]

15. Gosper, R.W. Harmonic Summation and Exponential gfs. 1996. Available online: http://mathworld.wolfram.com/ HarmonicNumber.html (accessed on 1 May 2019).

16. Rochowicz, J.A., Jr. Harmonic Numbers: Insights, Approximations and Applications. Spreadsheets Educ. 2015, 8, 4633.

17. Weisstein, E.W. CRC Concise Encyclopedia of Mathematics; Chapman and Hall/CRC: Boca Raton, FL, USA, 2003; p. 3115, ISBN 158488-347-2. 
18. Licciardi, S. Umbral Calculus, a Different Mathematical Language. Ph.D. Thesis, Department of Mathematics and Computer Sciences, XXIX Cycle, University of Catania, Catania, Italy, 2018.

19. Roman, S. The Umbral Calculus; Dover Publications: New York, NY, USA, 2005.

20. Choi, J.; Anderson, P.J.; Srivastava, H.M. Some $q$-extensions of the Apostol-Bernoulli and the Apostol-Euler polynomials of order $n$, and the multiple Hurwitz zeta function. Appl. Math. Comput. 2008, 199, 723-737. [CrossRef]

21. Alzer, H.; Choi, J. Four parametric linear Euler sums. J. Math. Anal. Appl. 2020, 484, 123661. [CrossRef]

22. Khan, N.; Aman, M.; Usman, T.; Choi, J. Legendre-Gould Hopper-Based Sheffer Polynomials and Operational Methods. Symmetry 2020, 12, 2051. [CrossRef]

23. Cvijović, D. The Dattoli-Srivastava Conjectures Concerning Generating Functions Involving the Harmonic Numbers. Appl. Math Comput. 2010, 215, 4040-4043. [CrossRef]

24. Dattoli, G. Generalized polynomials, operational identities and their applications. J. Comput. Appl. Math. 2000, 118, 111-123. [CrossRef]

25. Dattoli, G.; Germano, B.; Licciardi, S.; Martinelli, M.R. Hermite Calculus in Modeling in Mathematics, Atlantis Transactions in Geometry; Gielis, J., Ricci, P., Tavkhelidze, I., Eds.; Atlantis Press: Paris, France; Springer: New York, NY, USA, 2017; Volume 2, pp. 43-52. 